\title{
Understanding of Patient Engagement and its Impact on Healthcare Systems
}

\author{
Sima Marzban* \\ Gillings School of Global Public Health, University of North Carolina, Chapel Hill, USA
}

*Corresponding author: Sima Marzban, Gillings School of Global Public Health, University of North Carolina, Chapel Hill, USA.
Received Date: December 08, 2020

Published Date: January 07, 2021

\begin{abstract}
COVID-19 pandemic draws stakeholders' attention to strategies highlighting the patients' potential role and responsibilities in improving healthcare outcomes. There has been a constant effort to inform and educate patients to be compliant and adhere to the pre-planned treatment pathways/protocols based on existing evidence, insurers' policies, and providers' preferences. But after-Covid healthcare market transition reveals the game-changing impact of patients' priorities and personal behaviors on how they respond to surrounding decisions. Facts support the crucial need to update patient-oriented paradigms like Patient Engagement (PE) that prefer valued, informed, heard, and activated patients to contribute to all aspects of care, including healthcare policies, and processes. The current healthcare industry perception of PE incompletely addresses the patients' rights to access and share health records, prescriptions, transparent prices, billings, and information sources to take responsibility and control over the care. This, while patients can influence not only their personal care but also the peer population policies for future services. On the other hand, patients' contribution to the service design and interventions helps us acknowledge diverse values and personalize the care process based on individual variables.
\end{abstract}

As a result, passive unilateral patient engagement efforts such as patient and family education should evolve to active two-ways hearing of the patient voices incorporating their needs and expectations in individual care planning as much as health systems design and development. This evolution will be built on two fundamental changes;

- How patient-centeredness and engagement is directed and objectified by payors, providers, and supplier industries,

- How accreditation and healthcare quality institutions measure, assess, and evaluate patient-oriented interactions and quality measures.

PE requires exploring clinical and non-clinical insights from patients' eyes in addition to other stakeholders to reduce the gaps between therapeutic systems' and patients' actual needs and preferences. 


\section{Introduction}

The healthcare industry has developed various patient-centered concepts during the last decades' journey of transformation. The path has begun with patient education, has continued with patient empowerment, targeted patient satisfaction, and, more recently, seeking patients' engagement to achieve better health outcomes. The Medical Institute (IOM) considers access to appropriate medical information and clinical knowledge to be a source of control over individual health decisions [1]; however, available information and education seem to be a part of the concept. Activating patients to the extent that they take responsibility and actions to prevent further disease progression and obtain better wellness status depends on approaches beyond patient education. Active patient participation guarantees better health outcomes, return on investment, and lower cost per quality as the ultimate consequences of informed and accountable adherence to the care plan and medications [2].

Obviously, patient-family issues such as shortcomings in social determinants of health, including health literacy, income, nutrition, and housing, cause patient engagement barriers [3]. That is why inequity drivers would negatively influence patients and families' capacity to take an active role in engagement. It looks imperative or crucial to know to what extent existing patient advocacy/ engagement programs or technological solutions have enabled and mobilized patients to play a crucial role in improving individual treatment or population outcomes. Eventually, how current engagement strategies are aligned with patients' expected results.

\section{Patients Role Evolution}

Patient engagement is primarily understood by the smooth flow of information between providers and patients to ensure timely access to the needed resources. To this end, technology developers have designed numerous versions of platforms facilitating appointments, documentation, monitoring, billing, and evaluation of clinical performance. Furthermore, artificial intelligence fulfills digital communication platforms through big clinical data analytics to predict future clinical trends. Achieved insights accommodate supplier industries, insurers, and clinical providers' intensity to obtain insights on the business trajectory and plan for proactive performance changes.

On a higher level of deliberate, patient engagement requires cultural views and an organizational atmosphere that values the patients as health right owners, efficient sources of improvement, and investment partners. In that situation, patient empowerment is presumed beneficial not only for patients and families but also for policy developers and clinical providers. Transformative thoughts and beliefs are needed to support patients for being an honest advocate and organizational partner who puts his/her health in our hands for the best of actions to achieve scientific, professional, and business goals. Intelligent organizations are sensitive to customer insights in which the user experience, behaviors, and decisions mirror the gaps in care and communication. With the above logic, mature healthcare organizations embed PE in policy, practice, management, and evaluations to function with patient-oriented cultural values and visionary leadership. Keeping patients active and influential to the clinical teams subsequent a chain of activities that bring "patient insights to tangible impacts."

\section{Patient Insights and Healthcare Improvement}

Traditionally, quality improvement efforts are designed to review clinical facts and interventions conducted, implemented, and evaluated by providers. Ignoring numerous studies indicating patient-driven insights provided from patient cognitive and behavioral sources excel improvement efforts to a more advancement [4]. Fortunately, Patient-Reported Outcome Measures PROMS notices the value of a patient's health condition comes directly from the patient mouth; still, it primarily focuses on medical aspects of the disease like pain, disease progress, and associated mental health. Most clinical data platforms are designed to capture, track, and save documents of clinical dimensions such as symptoms, signs, appointments, billing process, diagnostics, and results [5]. Payors and providers recognize the included data categories. Whereas patients often experience uncertain journeys and life quality challenges caused by economic concerns, information gaps, decision requirements, non-transparent prices, employment issues, cultural conflicts, and family conditions because of disease. The challenging situations that influence the healing process somehow more influential than medications or clinical interventions.

\section{Acknowledgement}

None.

\section{Conflict of Interest}

Author declare no conflict of interest.

\section{References}

1. Cryer D (2011) Defining Patient Engagement: CFAH.

2. Barry MJ, Edgman Levitan S, Billingham V (2012) Shared Decision Making- The Pinnacle of Patient-Centered Care. N Engl J Med 366(9): 780-781.

3. Gagliardi AR., Lemieux-Charles L, Brown AD, Sullivan T, Goel V (2008) Barriers to patient involvement in health service planning and evaluation: an exploratory study. Patient Educ Couns 70(2): 234-241.

4. Prey JE, Woollen J, Wilcox L, Sackeim AD, Hripcsak G, et al. (2014) Patient engagement in the inpatient setting: a systematic review. J Am Med Inform Assoc 21(4): 742-750.

5. Weiner JP (2012) Doctor-patient communication in the e-health era. Isr J Health Policy Res 1(1): 33 